\title{
Mathematical Modelling and Simulation of Band Pass Filters using the Floating Admittance Matrix Method
}

\author{
SANJAY KUMAR ROY \\ Ph.D. Scholar, Lovely \\ Professional University and Head \\ (Power Distribution), OMQ \\ Division, \\ TATA Steel Ltd, INDIA.
}

\author{
KAMAL KUMAR SHARMA \\ Professor, Electronics \& \\ Communication Engineering, \\ Lovely Professional University, \\ Phagwara, Punjab, INDIA
}

\author{
BRAHMADEO PRASAD SINGH \\ Professor Adjunct, Electronics \& \\ Communication Engineering, Netaji \\ Subhas University of Technology, \\ New Delhi, INDIA
}

\author{
CHERRY BHARGAVA \\ Associate Professor, Computer \\ Science and Engineering, \\ Symbiosis Institute of Technology, \\ Pune, INDIA
}

\begin{abstract}
This article aims to develop a band pass filter's mathematical model using the Floating Admittance Matrix (FAM) method. The use of the conventional methods of analysis based KCL, KVL, Thevenin's, Norton's depends on the type of the particular circuit. The proposed mathematical modeling using the floating admittance matrix method is unique, and the same can be used for all types of circuits. This method uses the partitioning technique for large network. The sum property of all the elements of any row or any column equal to zero provides the assurance to proceed further for analysis or re-observe the very first equation. This saves time and energy. The FAM method presented here is so simple that anybody with slight knowledge of electronics but understating the matrix maneuvering, can analyze any circuit to derive all types of transfer functions. The mathematical modelling using the FAM method provides leverage to the designer to comfortably adjust their design at any stage of analysis. These statements provide compelling reasons for the adoption of the proposed process and demonstrate its benefits.
\end{abstract}

Keywords: Band-Pass Filters, Active Filters, Passive Filters, Mathematical model, Floating Admittance Matrix.

Received: January 4, 2021. Revised: July 19, 2021. Accepted: August 3, 2021. Published: August 16, 2021.

\section{Introduction}

Active and passive filters are building blocks of any electronic and communication systems that can alter or change the amplitude and/or phase characteristics of a signal. Active and passive filter are linear circuits that reshapes the unwanted signals and distortion from the contaminated input signal. Ideally, active and passive filter alters the various frequency components associated with amplitudes and the phase. The frequency-domain behavior of signals defines a particular type of filter. The transfer function of any filter is simply the Laplace transform of the output signal to the Input Signal. Filter Circuit, which consists of any active devices (transistors) and Op. amps, in addition to resistors, inductors, and capacitors, is called the active filter. On the other hand, a filter circuit designed with passive components only such as resistors, capacitors, and inductors, is called a passive filter. The operating frequency range of the filter is used to determine the electronic components in designing the circuit. Hence, the filter can also be further categorized based on the operating frequency of a particular circuit. In signal processing, a band-stop filter or band-reject or notch filter is a circuit that passes almost all frequencies unchanged but attenuates those in a specific range to very low levels. It is working just opposite to a band pass filter. The notch filter is designed to stop a very small band of frequencies, called a band-stop filter with a very narrow stop-band. Other names of this type of filters include "band limit filter," "T-notch filter," "band-elimination filter," and "band-reject filter."

\section{Methodology}

The conventional method of analysis uses one of the most suitable methods from KCL, KVL, Thevenin's, Norton's, etc., as per the suitability for a particular circuit, whether active or passive. The proposed floating admittance matrix method is unique, and the same can be used for all types of circuits. The complicated network can take the advantage of the matrix partitioning technique. The sum property of all elements of any row or any column elements equal to zero provides confidence to proceed further for analysis or re-observe the circuit at the very first equation to save time and energy.

The planar spiral type of inductor occupies more space and is associated with the low-quality factor; which is detrimental to be used in the filter circuit design. Because of this reason, Faruqe et al. [1] used simulated inductance to achieve a high-quality factor for RF applications. This paper presents a comparative analysis of active inductor design for high-quality factors at high-frequency applications based on 
the active inductor topologies. Active inductor-based circuits are commonly used in integrated circuits where the inductor's quality factor dominates the performance of the designed circuits. As the planner spiral inductor occupies a large area and shows a low-quality factor, an active inductor is an excellent option to overcome the spiral inductors' drawbacks. This paper summarizes the analysis and simulation results to select one of the best active inductor topologies to generate high-quality factors for high-frequency applications.

The use of shunt filters for better system performance Shady et al. [2] was discussed in the multi constraints belonging to the domain of multi-objective functions. The main aim here is to minimize total harmonic distortion of the load voltage, supply line, and cost minimization.

Shady et al. [3] demonstrates the optimal design technique to effectively utilize the cables and transformers for harmonically contaminated voltages and currents, accounting for the frequency-dependent loss of power. The method is especially suitable for a higher current carrying capacity of load.

It is shown that HP filters have dual properties than LP filters in the sense of sensitivity. Among various topologies of the BP filters, the best topology was demonstrated in this article.

The knowledge of the transfer function's characterization gives enough information to decide that the correct functioning of the circuit will be achieved. The variations or changes in the input and output impedances, power supply coupling and uncoupling, variation in the circuit components, and the other dynamic behaviors in the filters' structure are essential parameters discussed in detail by Bogdan [4].

Sargar et al. [5] suggests in-depth modelling and design procedure of LCR filter, primarily for inverter design used in alternative green energy sources such as a solar system. The MATLAB simulation of PV cell, DC-DC boost converter, and inverter with LCLR filter was also included in the paper.

The heuristic method is supposed to be the easiest and the best for the single tuned passive filters' optimized solution tapping of its essential parameters; which is very difficult. Such techniques provide a reasonable solution in a short time as it uses fewer iterations. The process called Response Surface Methodology (RSM) proposed by Sakar et al [6] was presented to solve issues of paper [5]. This approach looks to

minimize the harmonic distortion in voltage and current of the circuit.

Mathematical modelling based on the admittance matrix model [7]-[9] uses older elements such as norators, and nullators. Papers [10]-[13] suggested modelling techniques for measuring different parameters using FAM of threeterminal active devices such as FETs and BJTs. The circuit synthesis using passive components (resistors, inductors, and capacitors) is discussed in detail [14]-[15].

Desensitization using impedance tapering is being used to design a class-3 circuit with negative feedback [16]-[17]. In the negative feedback loops, the RC-section impedance is scaled upward from the driving source to the negative terminal of the amplifier input.

Frequency-selective circuits pass the signals of a range of frequencies through it without alteration in the input signal's magnitude. The pass-band filter passes the signal without alteration in the magnitude of the input signal to the output port. However, the magnitude of the input signal passed is reduced drastically, ideally to zero value, outside the pass band. The input currents of such circuits are of no significance, so their transfer functions are not examined.

In the subsequent subsection, we will take up some of all types of RC filters, and analyze one by one, using the floating admittance matrix approach (FAM).

\section{First-Type Bridge T-Network}

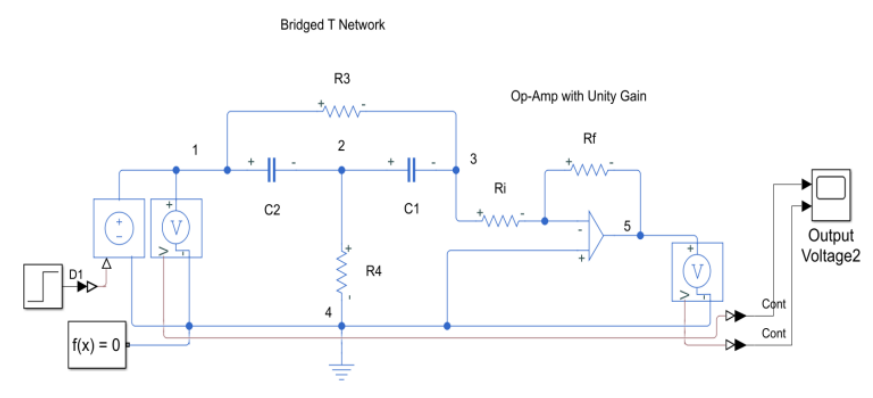

Fig. 1 First-Type Bridge -T Network

The floating admittance matrix of the bridge-T network without Op. Amp in Fig. 1.is written [10]-[13] as;

$\left[\begin{array}{cccc}1 & 2 & 3 & 4 \\ G_{3}+s C_{2} & -s C_{2} & -G_{3} & 0 \\ -s C_{2} & s C_{1}+s C_{2}+G_{4} & -s C_{1} & -G_{4} \\ -G_{3} & -s C_{1} & G_{3}+s C_{1} & 0 \\ 0 & -G_{4} & 0 & G_{4}\end{array}\right]\left[\begin{array}{l}1 \\ 1 \\ 2 \\ 3 \\ 4\end{array}\right]$

The open-circuit voltage transfer function between terminals $3 \& 4$ and $1 \& 4$ can be expressed [10]-[13] as;

$\left.A_{v}\right|_{14} ^{34}=\operatorname{sgn}(3-4) \operatorname{sgn}(1-4)(-1)^{12} \frac{\left|Y_{34}^{14}\right|}{\left|Y_{14}^{14}\right|}$

$\left|Y_{34}^{14}\right|=\left|\begin{array}{cc}-s C_{2} & s C_{1}+s C_{2}+G_{4} \\ -G_{3} & -s C_{1}\end{array}\right|$

$=s^{2}+s \frac{\left(C_{1}+C_{2}\right) G_{3}}{C_{1} C_{2}}+\frac{G_{3} G_{4}}{C_{1} C_{2}}$

$\left|Y_{14}^{14}\right|=\left|\begin{array}{cc}s C_{1}+s C_{2}+G_{4} & -s C_{1} \\ -s C_{1} & G_{3}+s C_{1}\end{array}\right|$

$=\left|\begin{array}{cc}s C_{2}+G_{4} & -s C_{1} \\ G_{3} & G_{3}+s C_{1}\end{array}\right|$

$=\left|\begin{array}{cc}s C_{2}+G_{3}+G_{4} & G_{3} \\ G_{3} & G_{3}+s C_{1}\end{array}\right|$

$=s^{2}+s \frac{\left\{\left(C_{2}+C_{1}\right) G_{3}+C_{1} G_{4}\right\}}{C_{1} C_{2}}+\frac{G_{3} G_{4}}{C_{1} C_{2}}$

$\left.A_{v}\right|_{14} ^{34}=\frac{s^{2}+s\left(\frac{1}{C_{1}}+\frac{1}{C_{2}}\right) \frac{1}{R_{3}}+\frac{1}{C_{1} C_{2} R_{3} R_{4}}}{s^{2}+s\left\{\left(\frac{1}{C_{1}}+\frac{1}{C_{2}}\right) \frac{1}{R_{3}}+\frac{1}{C_{2} R_{4}}\right\}+\frac{1}{C_{1} C_{2} R_{3} R_{4}}}$ 
$=\frac{s^{2}+s a+\omega_{0}^{2}}{s^{2}+s b+\omega_{0}^{2}}=\frac{s^{2}+s \frac{\omega_{0}}{Q}+\omega_{0}^{2}}{s^{2}+s \frac{\omega_{01}}{Q}+\omega_{0}^{2}}$

Since, $\left.\beta(s)\right|_{34} ^{14}=\frac{1}{\left.A_{v}\right|_{14} ^{34}}$

Considering inverting amplifier include in the circuit of Fig.

1 , the overall voltage transfer function is;

$\left.A_{v}\right|_{14} ^{54}=\frac{v_{54}}{v_{14}}=\frac{v_{34}}{v_{14}} x \frac{v_{54}}{v_{34}}$

$=\frac{s^{2}+s \frac{\omega_{0}}{Q}+\omega_{0}^{2}}{s^{2}+s \frac{\omega_{0}}{Q_{1}}+\omega_{0}^{2}} x\left(-\frac{R_{F}}{R_{i}}\right)=-\frac{s^{2}+s \frac{\omega_{0}}{Q}+\omega_{0}^{2}}{s^{2}+s \frac{\omega_{0}}{Q_{1}}+\omega_{0}^{2}}$ for $\mathrm{R}_{\mathrm{F}}=\mathrm{R}_{\mathrm{i}}$

The transmission zero in Eq. (4) is similar to the poles of the closed-loop circuit of Fig. 1. Hence, the transmission zero forms the polynomial as;

$$
\begin{aligned}
& s^{2}+s \frac{\omega_{0}}{Q}+\omega_{O}^{2}=s^{2}+s a+\omega_{O}^{2} \\
& =s^{2}+s\left(\frac{1}{C_{1}}+\frac{1}{C_{2}}\right) \frac{1}{R_{3}}+\frac{1}{C_{1} C_{2} R_{3} R_{4}} \\
& \omega_{O}^{2}=\frac{1}{C_{1} C_{2} R_{3} R_{4}}, Q=\frac{\omega_{0}}{a}=\frac{\omega_{0}}{\left(\frac{1}{C_{1}}+\frac{1}{C_{2}}\right) \frac{1}{R_{3}}}=\frac{\omega_{0}}{\left\{\frac{C_{1}+C_{2}}{C_{1} C_{2} R_{3}}\right\}} \\
& =\frac{\sqrt{C_{1} C_{2} R_{3} / R_{4}}}{C_{1+} C_{2}}
\end{aligned}
$$$$
\text { For, } \mathrm{R}_{2}=\mathrm{R}_{4}=\mathrm{R} \text {, and } \mathrm{C}_{1}=\mathrm{C}_{2}=\mathrm{C}, \mathrm{Q}=0.5
$$

The characteristic equation for the pole is;

$s^{2}+s \frac{\omega_{0}}{Q_{1}}+\omega_{0}^{2}=s^{2}+s b+\omega_{O}^{2}$

where, $b=\left\{\left(\frac{1}{C_{1}}+\frac{1}{C_{2}}\right) \frac{1}{R_{3}}+\frac{1}{C_{2} R_{4}}\right\}=\frac{2}{R C}+\frac{1}{R C}=\frac{3}{R C}$, and $\omega_{0}=\frac{1}{R C}, \frac{\omega_{0}}{Q_{1}}=\frac{3}{R C}=3 \omega_{0}, Q_{1}=\frac{1}{3}$

The characteristic equation for the pole is now expressed as;

$$
s^{2}+3 \omega_{0} s+\omega_{0}^{2}
$$

Though there is no need to find out the current transfer ratio, we include both current transfer and power transfer ratios for academic purposes. The current transfer function between terminals $3 \& 4$ and $1 \& 4$ can be expressed [13] as;

$$
\begin{aligned}
& \left.A_{i}\right|_{14} ^{34}=\operatorname{sgn}(3-4) \operatorname{sgn}(1-4)(-1)^{12} \frac{\left|Y_{34}^{14}\right|}{\left|Y_{4}^{4}\right|} G_{L} \\
& \left|Y_{4}^{4}\right|=\left|\begin{array}{ccc}
G_{3}+s C_{2} & -s C_{2} & -G_{3} \\
-s C_{2} & s C_{1}+s C_{2}+G_{4} & -s C_{1} \\
-G_{3} & -s C_{1} & G_{3}+s C_{1}
\end{array}\right| \\
& =\left|\begin{array}{ccc}
G_{3}+s C_{2} & 0 & -G_{3} \\
0 & G_{4} & 0 \\
-G_{3} & 0 & G_{3}+s C_{1}
\end{array}\right| \\
& =G_{4}\left\{\left(G_{3}+s C_{2}\right)\left(G_{3}+s C_{1}\right)-G_{3}^{2}\right\} \\
& =G_{4} s\left\{s C_{1} C_{2}+G_{3}\left(C_{1}+C_{2}\right)\right\} \\
& \left.A_{i}\right|_{14} ^{34}=\frac{s^{2} C_{1} C_{2}+s\left(C_{1}+C_{2}\right) G_{3}+G_{3} G_{4}}{G_{4} s\left\{s C_{1} C_{2}+G_{3}\left(C_{1}+C_{2}\right)\right\}} G_{L}
\end{aligned}
$$

The power transfer function between terminals $3 \& 4$ and $1 \&$ 4 can be expressed as;

$\left.A_{p}\right|_{14} ^{34}=\left.\left.A_{v}\right|_{14} ^{34} x A_{i}\right|_{14} ^{34}$

$$
\begin{aligned}
\left.A_{p}\right|_{14} ^{34}= & \left\{\frac{s^{2} C_{1} C_{2}+s\left(C_{1}+C_{2}\right) G_{3}+G_{3} G_{4}}{\left.s^{2} C_{1} C_{2}(33)\left(C_{2}+C_{1}\right) G_{3}+C_{1} G_{4}\right\}+G_{3} G_{4}}\right\} \\
& \left\{\frac{s^{2} C_{1} C_{2}+s\left(C_{1}+C_{2}\right) G_{3}+G_{3} G_{4}}{G_{4} s\left\{s C_{1} C_{2}+G_{3}\left(C_{1}+C_{2}\right)\right\}} G_{L}\right\}
\end{aligned}
$$

\section{Experimental Verification}

The MATLAB program for the bridge-T network in Fig. 1 and its Bode plot is shown in Fig. 2. It depicts the plot for amplitude and phase variation with reference to frequency. The assumed value of resistances and capacitances are indicated in the program below.

r3 $=1500 ; \mathrm{r} 4=1500$;

$\mathrm{c} 1=1000 \mathrm{e}-6 ; \mathrm{c} 2=2000 \mathrm{e}-6$;

$\mathrm{A}=(1 / \mathrm{c} 1+1 / \mathrm{c} 2) / \mathrm{r} 3$;

$\mathrm{B}=1 / \mathrm{c} 1 * 1 / \mathrm{c} 2 * 1 / \mathrm{r} 3 * 1 / \mathrm{r} 4$

$\mathrm{C}=1 / \mathrm{c} 1 * 1 / \mathrm{c} 2 * 1 / \mathrm{r} 3 * 1 / \mathrm{r} 4$

$\mathrm{D}=1 / \mathrm{c} 1 * 1 / \mathrm{c} 2 * 1 / \mathrm{r} 3 * 1 / \mathrm{r} 4$

num $=[1$ A B $] ;$ (אe) = [1 C D $]$;

$\mathrm{GP}=\mathrm{tf}($ num,den);

bode(GP);

$$
\mathrm{GP}=\frac{\mathrm{s}^{\wedge} 2+\mathrm{s}+0.2222}{\mathrm{~s}^{\wedge} 2+0.2222 \mathrm{~s}+0.2222}
$$

The plot of the above-described continuous time domain voltage transfer function without and with an inverter, shown in Figs 2 and 3, respectively, indicate phase difference of 180 degrees.

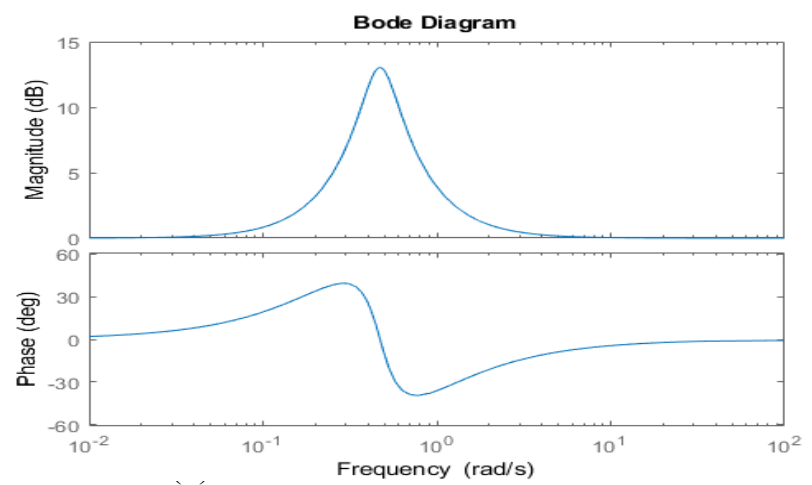

Fig.2 Plot of Bridge Network transfer function with frequency without Op Amp.

The MATLAB program for the bridge-T network in Fig. 1 and its Bode plot including Op Amp is shown in Fig. 3.

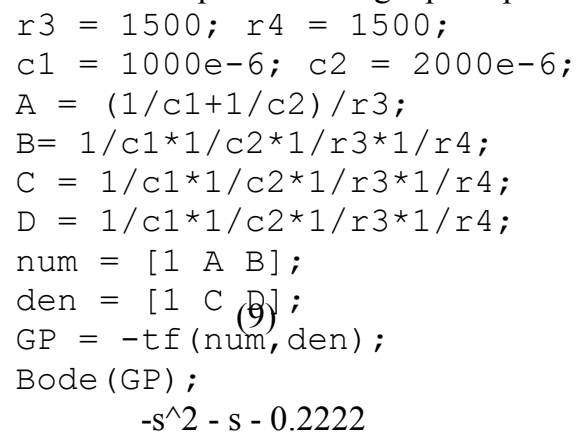

$\mathrm{GP}=$

This is the continuous-time domain transfer function. 


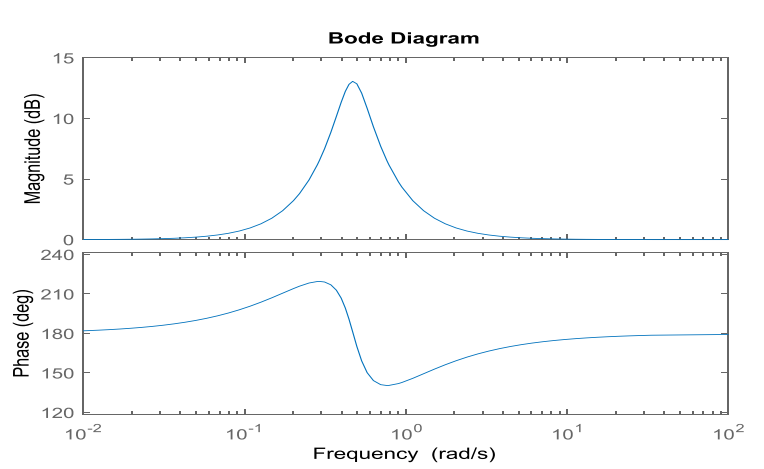

Fig. 3 Plot of Bridge Network transfer function including Op-

Amp w.r.t frequency.

The input impedance between terminals $1 \& 4$ in Fig. 1 is expressed [10]-[13] as:

$Z_{\text {in }}=Z_{14}={\frac{\left|Y_{14}^{14}\right|}{\left|Y_{4}^{4}\right|}}_{g_{s}=0}$

$\left|Y_{4}^{4}\right|=\left|\begin{array}{ccc}G_{3}+s C_{2} & -s C_{2} & -G_{3} \\ -s C_{2} & s C_{1}+s C_{2}+G_{4} & -s C_{1} \\ -G_{3} & -s C_{1} & G_{3}+s C_{1}\end{array}\right|$

$=\left|\begin{array}{ccc}G_{3}+s C_{2} & 0 & -G_{3} \\ -s C_{2} & G_{4} & -s C_{1} \\ -G_{3} & 0 & G_{3}+s C_{1}\end{array}\right|=G_{4}\left|\begin{array}{cc}G_{3}+s C_{2} & -G_{3} \\ -G_{3} & G_{3}+s C_{1}\end{array}\right|$

$=G_{4}\left|\begin{array}{cc}s C_{2} & -G_{3} \\ s C_{1} & G_{3}+s C_{1}\end{array}\right|=G_{4}\left\{s^{2} C_{1} C_{2}+s\left(C_{1}+C_{2}\right) G_{3}\right\}$

$=G_{4} s\left\{s C_{1} C_{2}+\left(C_{1}+C_{2}\right) G_{3}\right\}$.

$Z_{\text {in }}=Z_{14}=\frac{\left|Y_{14}^{14}\right|}{\left|Y_{4}^{4}\right|_{g_{s}=0}}$

$=\frac{s^{2} C_{1} C_{2}+s\left\{\left(C_{1}+C_{2}\right) G_{3}+C_{1} G_{4}\right\}+G_{3} G_{4}}{G_{4} s\left\{C_{1} C_{2}+\left(C_{1}+C_{2}\right) G_{3}\right\}}$

$=\frac{1}{G_{4}}+\frac{s C_{1}+G_{3}}{s C_{2}\left(s C_{1}+G_{3}\right)+C_{1} G_{3}}$

$=\frac{1}{G_{4}}+\frac{1}{s C_{2}+\frac{s C_{1} G_{3}}{s C_{1}+G_{3}}}$

This Eq. (14) of input impedance represents the network of the form shown in Fig. 4.

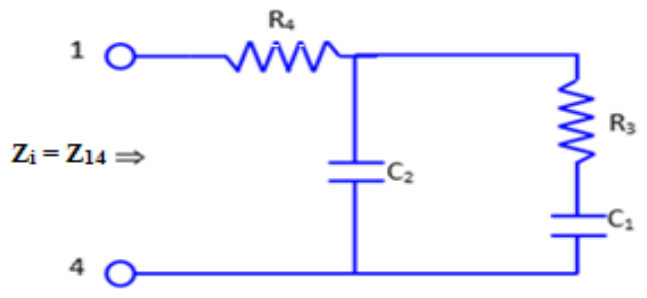

Fig. 4 input impedance representation

The output impedance between terminals $3 \& 4$ of Fig. 1 can be expressed [10]-[13] as;

$R_{o}=R_{34}=\frac{\left|Y_{34}^{34}\right|}{\left|Y_{4}^{4}\right|}{ }_{G_{L}=0}$

$\left|Y_{34}^{34}\right|=\left|\begin{array}{cc}G_{3}+s C_{2} & -s C_{2} \\ -s C_{2} & s C_{1}+s C_{2}+G_{4}\end{array}\right|$
$=\left|\begin{array}{cc}G_{3}+s C_{2} & G_{3} \\ -s C_{2} & s C_{1}+G_{4}\end{array}\right|$

$=s^{2} C_{1} C_{2}+s\left(C_{1}+C_{2}\right) G_{3}+s C_{2} G_{4}+G_{3} G_{4}$

$R_{o}=R_{34}=\frac{\left|Y_{34}^{34}\right|}{\left|Y_{4}^{4}\right|}{ }_{G_{L}=0}$

$=\frac{s^{2} C_{1} C_{2}+s\left(C_{1}+C_{2}\right) G_{3}+s C_{2} G_{4}+G_{3} G_{4}}{G_{4}\left\{s^{2} C_{1} C_{2}+s\left(C_{1}+C_{2}\right) G_{3}\right\}}$

$=\frac{1}{G_{4}}+\frac{\left(s C_{2}+G_{3}\right)}{s\left(s C_{2}+G_{3}\right) C_{1}+s C_{2} G_{3}}=\frac{1}{G_{4}}+\frac{1}{s C_{1}+\frac{s C_{2} G_{3}}{s C_{2}+G_{3}}}$

The circuit of output impedance in Fig. 5 represents Eq. (17).

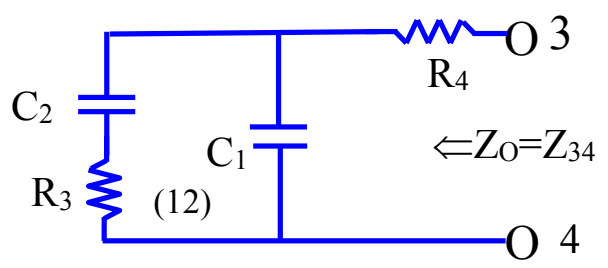

Fig.5 Output impedance representation

\section{Second Form of Bridge -T Network}

Figure 6 shows the dual (second type) form of the bridge-T network for realizing the same type of transmission zeros.

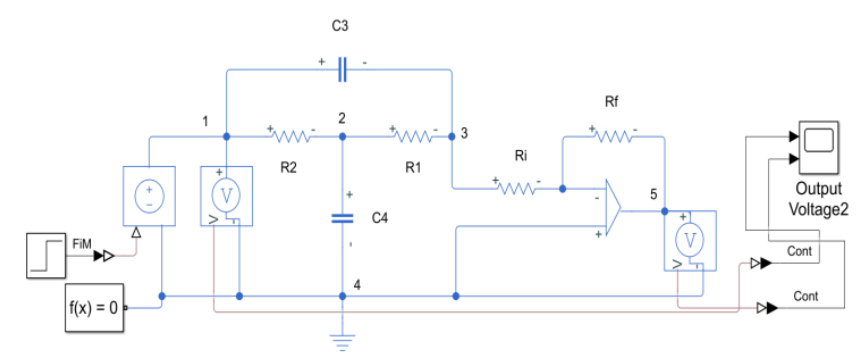

Fig. 6 Second-Type Bridge-T Network

(14)

The floating admittance matrix for Fig. 6 is expressed [10]-

[13] as;

$\left[\begin{array}{cccc}1 & 2 & 3 & 4 \\ G_{2}+s C_{3} & -G_{2} & -s C_{3} & 0 \\ -G_{2} & G_{1}+G_{2}+s C_{4} & -G_{1} & -s C_{4} \\ -s C_{3} & -G_{1} & G_{1}+s C_{3} & 0 \\ 0 & -s C_{4} & 0 & s C_{4}\end{array}\right]\left[\begin{array}{l}1 \\ 2 \\ 3 \\ 4\end{array}\right]$

The open-circuit voltage transfer function between terminals $3 \& 4$ and $1 \& 4$ of Fig. 6 can be expressed [10]-[13] as;

$\left.A_{v}\right|_{14} ^{34}=\operatorname{sgn}(3-4) \operatorname{sgn}(1-4)(-1)^{12} \frac{\left|Y_{34}^{14}\right|}{\left|Y_{14}^{14}\right|}$

$\left|Y_{34}^{14}\right|=\left|\begin{array}{cc}-G_{2} & G_{1}+G_{2}+s C_{4} \\ -s C_{3} & -G_{1}\end{array}\right|$

$=G_{1} G_{2}+s\left(G_{1}+G_{2}\right) C_{3}+s^{2} C_{3} C_{4}$

$=s^{2}+s \frac{\left.\left(G_{1}+G_{2}\right) C_{\xi}\right)}{C_{3} C_{4}}+\frac{G_{1} G_{2}}{C_{3} C_{4}}$

$\left|Y_{14}^{14}\right|=\left|\begin{array}{cc}G_{1}+G_{2}+s C_{4} & -G_{1} \\ -G_{1} & G_{1}+s C_{3}\end{array}\right|$ 
$=\left|\begin{array}{cc}G_{2}+s C_{4} & -G_{1} \\ s C_{3} & G_{1}+s C_{3}\end{array}\right|$

$=s^{2} C_{3} C_{4}+s\left\{C_{3}\left(G_{1}+G_{2}\right)+C_{4} G_{1}\right\}+G_{1} G_{2}$.

$\left.A_{v}\right|_{14} ^{34}=\frac{\left|Y_{34}^{14}\right|}{\left|Y_{14}^{14}\right|}=\frac{s^{2}+s \frac{\left(G_{1}+G_{2}\right) C_{3}}{C_{3} C_{4}}+\frac{G_{1} G_{2}}{C_{3} C_{4}}}{s^{2}+s \frac{\left\{C_{3}\left(G_{1}+G_{2}\right)+C_{4} G_{1}\right\}}{C_{3} C_{4}}+\frac{G_{1} G_{2}}{C_{3} C_{4}}}$

$=\frac{s^{2}+s\left(\frac{1}{R_{1}}+\frac{1}{R_{2}}\right) \frac{1}{C_{4}}+\frac{1}{C_{3} C_{4} R_{1} R_{2}}}{s^{2}+s\left\{\left(\frac{1}{R_{1}}+\frac{1}{R_{2}}\right) \frac{1}{C_{4}}+\frac{1}{R_{1} C_{3}}\right\}+\frac{1}{C_{3} C_{4} R_{1} R_{2}}}=\frac{s^{2}+s \frac{\omega_{0}}{Q}+\omega_{0}^{2}}{s^{2}+s \frac{\omega_{0}}{Q_{1}}+\omega_{0}^{2}}$

The overall voltage transfer function between terminals $5 \& 4$ and $1 \& 4$ including the Op. Amp is;

$\left.A_{v}\right|_{14} ^{54}=\frac{v_{54}}{v_{14}}=\frac{v_{34}}{v_{14}} x \frac{v_{54}}{v_{34}}=\frac{s^{2}+s \frac{\omega_{0}}{Q}+\omega_{0}^{2}}{s^{2}+s \frac{\omega_{0}}{Q_{1}}+\omega_{0}^{2}} x\left(-\frac{R_{F}}{R_{i}}\right)=-\frac{s^{2}+s \frac{\omega_{0}}{Q}+\omega_{0}^{2}}{s^{2}+s \frac{\omega_{0}}{Q_{1}}+\omega_{0}^{2}}$

for $\mathrm{R}_{\mathrm{F}}=\mathrm{R}_{\mathrm{i}}$

In Eq. (21), the transmission zero is similar to the poles of the closed-loop circuit of Fig. 6. Hence, the transmission zero is expressed as;

$$
\begin{aligned}
& s^{2}+s \frac{\omega_{0}}{Q}+\omega_{0}^{2}=s^{2}+a s+\omega_{0}^{2} \\
& =s^{2}+s\left(\frac{1}{R_{1}}+\frac{1}{R_{2}}\right) \frac{1}{C_{4}}+\frac{1}{C_{3} C_{4} R_{1} R_{2}} \\
& \text { here, } \omega_{0}^{2}=\frac{1}{C_{3} C_{4} R_{1} R_{2}} \text { and } \\
& Q=\frac{\omega_{0}}{a}=\sqrt{\frac{1}{C_{3} C_{4} R_{1} R_{2}}} x \frac{1}{\frac{\left(G_{1}+G_{2}\right)}{C_{4}}}=\frac{\sqrt{R_{1} R_{2} C_{4} / C_{3}}}{R_{1}+R_{2}}
\end{aligned}
$$

For, $\mathrm{C}_{3}=\mathrm{C}_{4}=\mathrm{C}$, and $\mathrm{R}_{1}=\mathrm{R}_{2}=\mathrm{R}, \omega_{\mathrm{o}}=1 / \mathrm{RC}, \mathrm{Q}=0.5$

The characteristic equation for poles is;

$$
s^{2}+s \frac{\omega_{0}}{Q_{1}}+\omega_{0}^{2}=s^{2}+b s+\omega_{0}^{2},
$$

where, $b=\left(\frac{1}{R_{1}}+\frac{1}{R_{2}}\right) \frac{1}{C_{4}}+\frac{1}{R_{1} C_{3}}=\frac{3}{R C}$

$\frac{\omega_{0}}{Q_{1}}=\frac{3}{R C}=3 \omega_{0}, Q_{1}=\frac{1}{3}$

The characteristic equation for poles is now expressed as;

$$
s^{2}+3 \omega_{0} s+\omega_{0}^{2}
$$

The current transfer function between terminals $3 \& 4$ and 1 $\& 4$ of Fig. 6 can be expressed [13] as;

$$
\begin{aligned}
& \left.A_{i}\right|_{14} ^{34}=\operatorname{sgn}(3-4) \operatorname{sgn}(1-4)(-1)^{12} \frac{\left|Y_{34}^{14}\right|}{\left|Y_{4}^{4}\right|} G_{L} \\
& \left|Y_{4}^{4}\right|=\left|\begin{array}{ccc}
G_{2}+s C_{3} & -G_{2} & -s C_{3} \\
-G_{2} & G_{1}+G_{2}+s C_{4} & -G_{1} \\
-s C_{3} & -G_{1} & G_{1}+s C_{3}
\end{array}\right| \\
& =\left|\begin{array}{ccc}
G_{2}+s C_{3} & 0 & -s C_{3} \\
0 & s C_{4} & 0 \\
-s C_{3} & 0 & G_{1}+s C_{3}
\end{array}\right| \\
& =s C_{4}\left|\begin{array}{cc}
G_{2}+s C_{3} & -s C_{3} \\
-s C_{3} & G_{1}+s C_{3}
\end{array}\right|=s C_{4}\left|\begin{array}{ll}
G_{2} & -s C_{3} \\
G_{1} & G_{1}+s C_{1}
\end{array}\right| \\
& =s C_{4}\left\{s C_{1} G_{2}+s C_{3} G_{1}+G_{1} G_{3}\right\} \\
& \left.A_{i}\right|_{14} ^{34}=\frac{\left|Y_{34}^{14}\right|}{\left|Y_{4}^{4}\right|} G_{L}=\frac{s^{2} C_{3} C_{4}+s\left(G_{1}+G_{2}\right) C_{3}+G_{1} G_{2}}{s C_{4}\left\{s C_{1} G_{2}+s C_{3} G_{1}+G_{1} G_{3}\right\}} G_{L}
\end{aligned}
$$

The power transfer function between terminals $3 \& 4$ and $1 \&$ 4 of Fig. 6 can be expressed as;

$\left.A_{p}\right|_{14} ^{34}=\left.\left.A_{v}\right|_{14} ^{34} x A_{i}\right|_{14} ^{34}$ $=\left\{\frac{s^{2} C_{3} C_{4}+s\left(G_{1}+G_{2}\right) C_{3}+G_{1} G_{2}}{s^{2} C_{3} C_{4}+s\left\{C_{3}\left(G_{1}+G_{2}\right)+C_{4} G_{1}\right\}+G_{1} G_{2}}\right\}\left\{\frac{s^{2} C_{3} C_{4}+s\left(G_{1}+G_{2}\right) C_{3}+G_{1} G_{2}}{s C_{4}\left\{s C_{1} G_{2}+s C_{3} G_{1}+G_{1} G_{3}\right\}} G_{L}\right\}$

\section{Experimental Verification}

The MATLAB program for the bridge-T network shown in Fig. 6 is given below and its Bode plot is shown in Fig. 7. The plot shows amplitude and phase variation with reference to frequency. $(2 \phi)$ he assumed value of resistances and capacitances are indicated in the program.

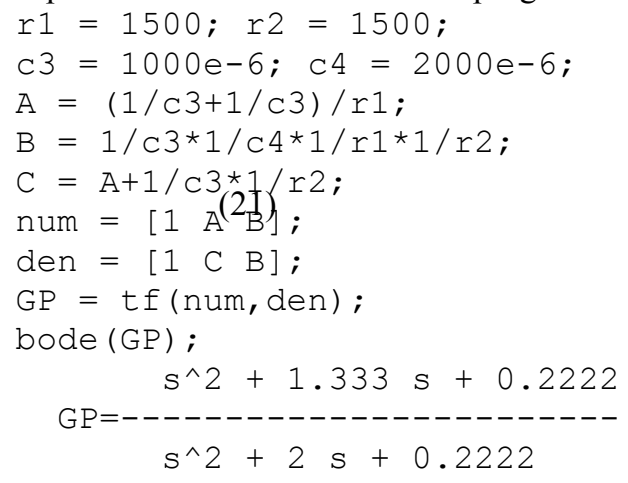

The continuous-time domain transfer function indicated by above equation is plotted in Fig.7.

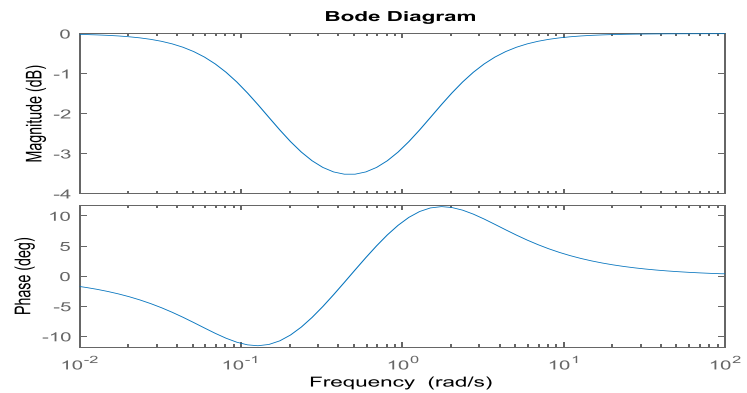

Fig. 7 Magnitude and phase plot of Bridge-T without OpAmp.

The MATLAB ${ }^{(24)}$ program for the bridge-T network shown in Fig. 6 is given below.

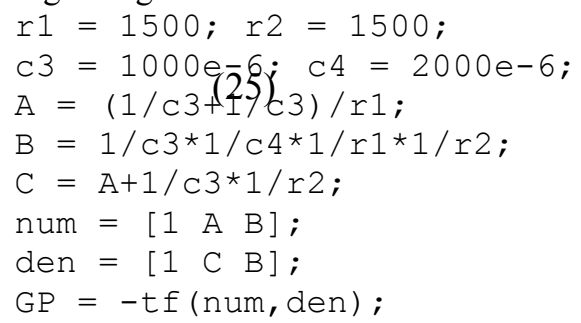

$$
\begin{gathered}
-s^{\wedge} 2-1.333 s-0.2222 \\
s^{\wedge} 2+2 s+0.2222
\end{gathered}
$$

The continuous-time domain transfer function indicated by above equation 2 fong with Op. Amp. in Fig. 6 is plotted in Fig. 8. 


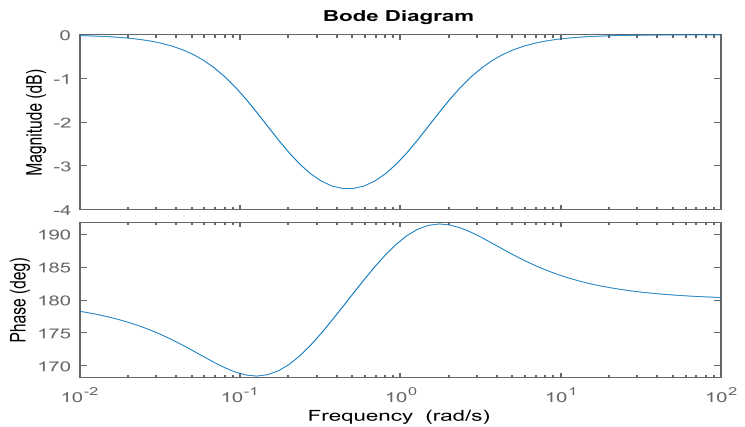

Fig. 8 Magnitude and phase plot of Bridge-T with Op-Amp

The input impedance between terminals $1 \& 4$ of Fig. 6 can be expressed [10]-[13] as;

$Z_{\text {in }}=Z_{14}=\frac{\left|Y_{14}^{14}\right|}{\left|Y_{4}^{4}\right|} g_{s}=0$

$\left|Y_{4}^{4}\right|=\left|\begin{array}{ccc}G_{2}+s C_{3} & -G_{2} & -s C_{3} \\ -G_{2} & G_{1}+G_{2}+s C_{4} & -G_{1} \\ -s C_{3} & -G_{1} & G_{1}+s C_{3}\end{array}\right|$

$=\left|\begin{array}{ccc}0 & s C_{4} & 0 \\ -G_{2} & s C_{4} & -G_{1} \\ -s C_{3} & 0 & G_{1}+s C_{3}\end{array}\right|$

$=-s C_{4}\left\{-\left(G_{1}+s C_{3}\right) G_{2}-s C_{3} G_{1}\right\}$

$=s C_{4}\left\{s\left(G_{1}+G_{2}\right) C_{3}+G_{2} G_{1}\right\}$

$Z_{\text {in }}=Z_{14}=\frac{\left|Y_{14}^{14}\right|}{\left|Y_{4}^{4}\right|}$

$=\frac{s^{2} C_{3} C_{4}+s\left\{C_{3}\left(G_{1}+G_{2}\right)+C_{4} G_{1}\right\}+G_{1} G_{2}}{s C_{4}\left\{s\left(G_{1}+G_{2}\right) C_{3}+G_{2} G_{1}\right\}}$

$=\frac{s\left\{C_{3}\left(G_{1}+G_{2}\right)\right\}+G_{1} G_{2}}{s C_{4}\left\{s\left(G_{1}+G_{2}\right) C_{3}+G_{2} G_{1}\right\}}+\frac{s^{2} C_{3} C_{4}+s C_{4} G_{1}}{s C_{4}\left\{s\left(G_{1}+G_{2}\right) C_{3}+G_{2} G_{1}\right\}}$

$\mathrm{Z}_{\mathrm{i}}=\mathrm{Z}_{14}=\frac{\mathrm{S}^{2} \mathrm{C}_{3} \mathrm{C}_{4}+\mathrm{S}\left\{\mathrm{C}_{3}\left(\mathrm{G}_{1}+\mathrm{G}_{2}\right)+\mathrm{C}_{4} \mathrm{G}_{1}\right\}+\mathrm{G}_{1} \mathrm{G}_{2}}{\mathrm{SC}_{4}\left\{\left(\mathrm{G}_{1}+\mathrm{G}_{2}\right) \mathrm{C}_{3}+\mathrm{G}_{1} \mathrm{G}_{2}\right\}}$

$=\frac{1}{\mathrm{SC}_{4}}+\frac{1}{\mathrm{G}_{2}+\frac{\mathrm{SC}_{3} \mathrm{G}_{1}}{\mathrm{SC}_{3}+\mathrm{G}_{1}}}$

$=\frac{1}{\mathrm{SC}_{4}}+\frac{1}{\mathrm{G}_{2}+\frac{\mathrm{SC}_{3} \mathrm{G}_{1}}{\mathrm{SC}_{3}+\mathrm{G}_{1}}}$

Figure 9 is the representation of the input impedance of Eq. (30).

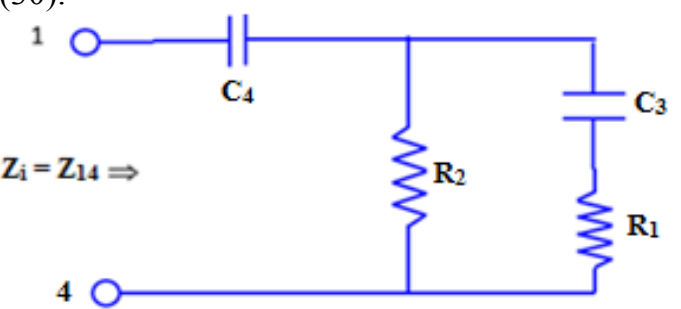

Fig.9 Input impedance representation

The output impedance of Fig. 6 between terminals $3 \& 4$ can be written [10]-[13] as;

$Z_{0}=Z_{34}=\frac{\left|Y_{34}^{34}\right|}{\left|Y_{4}^{4}\right|} G_{L}=0$

$\left|Y_{34}^{34}\right|=\left|\begin{array}{lc}G_{2}+S_{3} & -G_{2} \\ -G_{2} & G_{1}+G_{2}+S_{4}\end{array}\right|$

$=\left(\mathrm{G}_{1}+\mathrm{G}_{2}\right) \mathrm{G}_{2}+\mathrm{G}_{2} \mathrm{SC}_{4}+\mathrm{SC}_{3}\left(\mathrm{G}_{1}+\mathrm{G}_{2}\right)+\mathrm{S}^{2} \mathrm{C}_{3} \mathrm{C}_{4}-\mathrm{G}_{2}^{2}$

$=\mathrm{S}^{2} \mathrm{C}_{3} \mathrm{C}_{4}+\mathrm{S}\left\{\mathrm{C}_{3}\left(\mathrm{G}_{1}+\mathrm{G}_{2}\right)+\mathrm{C}_{4} \mathrm{G}_{2}\right\}+\mathrm{G}_{1} \mathrm{G}_{2}$

$\mathrm{Z}_{\mathrm{o}}=\mathrm{Z}_{34}$

$=\frac{\mathrm{S}^{2} \mathrm{C}_{3} \mathrm{C}_{4}+\mathrm{S}\left\{\mathrm{C}_{3}\left(\mathrm{G}_{1}+\mathrm{G}_{2}\right)+\mathrm{C}_{4} \mathrm{G}_{2}\right\}+\mathrm{G}_{1} \mathrm{G}_{2}}{\mathrm{SC}_{4}\left\{\mathrm{SC}_{3}\left(\mathrm{G}_{1}+\mathrm{G}_{2}\right)+\mathrm{G}_{1} \mathrm{G}_{2}\right\}}$

$=\frac{1}{\mathrm{SC}_{4}}+\frac{\left(2 \mathrm{SC}_{4}\left(\mathrm{SC}_{3}+\mathrm{G}_{2}\right)\right.}{\mathrm{SC}_{4}\left\{\mathrm{SC}_{3}\left(\mathrm{G}_{1}+\mathrm{G}_{2}\right)+\mathrm{G}_{1} \mathrm{G}_{2}\right\}}$

$=\frac{1}{\mathrm{SC}_{4}}+\frac{1}{\mathrm{G}_{1}+\frac{\mathrm{SC}_{3} \mathrm{G}_{2}}{\mathrm{SC}_{3}+\mathrm{G}_{2}}}$

Figure 10 shows the circuit representation of the output impedance as per Eq. (33).

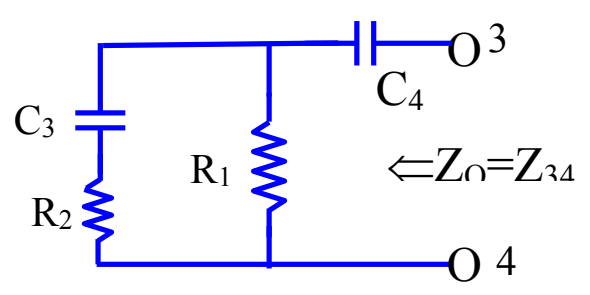

Fig.10 Output impedance representation

\section{Results and Discussions}

The plots in Figs. 2, 3, 7, and 8 corroborate the theoretical results obtain. The FAM mathematical model presented here is so simple that anybody with slight knowledge of electronics devices but understanding matrix maneuvering can analyse the circuits to derive all transfer functions provided the parmeters of devices are known to him/ her. The analysis and then designing any circuit using the floating admittance matrix model is based on pure mathematical maneuvering of matrix elements. The transfer functions are defined as min(301') ratio with proper signs, called cofactors of first and or second order. The mathematical modelling using the FAM approach provides leverage to the designer to comfortably adjust their design style at any analysis stage.

\section{Conclusions}

The modelling and simulation of bridge -T network has been carried out using MATLAB's Simulink system environment. This paper provides simulated and numerical validation of two forms of bridge -T network used for band pass filter. The MATLAB program developed for the transfer function for both types of the RC bridge-T network are plotted in the form 
of magnitude and phase w.r.t. frequency. The input and output impedances are derived and drawn using the FAM technique.

\section{References:}

[1] Omar Faruqe, Md. M. M. Saikat, Md. A. K. Bulbul and Md. Tawfiq Amin: "Comparative Analysis and Simulation of Active Inductors for RF Applications in $90 \mathrm{~nm}$ CMOS" 2017 3rd International Conference on Electrical Information and Communication Technology (EICT), 7-9 December 2017, Khulna, Bangladesh.

[2] H.E, Shady Abdel Aleem, Mohamed T. Elmathana and Ahmed F. Zobaa: "Different Design Approaches of Shunt Passive Harmonic Filters Based on IEEE Std. 519-1992 and IEEE Std. 182002". Recent Patents on Electrical \& Electronic Engineering 2013, 6, 68-75.

[3] H.E, Shady Abdel Aleem, Murat Erhan Balci, Selcuk Sakar: "Utilization of cables and transformers using passive filters for non-linear loads" Electrical Power and Energy Systems 71 (2015) 344-350.

[4] M. Bogdan, M. Panu: "Lab View Modeling and simulation of the Low pass and High Pass analog filters". 2015 13th International Conference on Engineering of Modern Electric Systems (EMES).

[5] D. S. Sargar, C. L. Bhattar. "LCLR filter design and modelling for harmonic mitigation in interconnected micro grid system." ISSN: 2319-1163 | ISSN: 23217308.

[6] Selcuk Sakar, Aslan Deniz Karaoglan, Murat Erhan Balci, Shady H. E. Abdel Aleem, Ahmed F. Zobaa.: "Optimal Design of Single-Tuned Passive Filters Using Response Surface Methodology". XII International School on Non-sinusoidal Currents and Compensation, ISNCC 2015, Łagów, Poland.

[7] Haigh, David G., and Paul M. Radmore. "Admittance matrix models for the nullor using limit variables and their application to circuit design", IEEE Transactions on Circuits and Systems I: Regular Papers 53, no. 10 (2006): 2214-2223.

[8] I. Vago, and E. Hollos. "Two-port models with nullators and norators", Periodica Polytechnica Electrical Engineering 17, no. 4 (1973): 301-309.

[9] Pragati Kumar, and Raj Senani. "Improved grounded-capacitor SRCO using only a single PFTFN", Analog Integrated Circuits and Signal Processing 50, no. 2 (2007): 147-149.

[10]B. P. Singh,"On demand realization of a frequencydependent negative resistance and an infinite input impedance" International Journal of Electronics Theoretical and Experimental, Taylor \& Francis, Vol.44, no. 3 (1978): 243-249.

[11]Wai-Kai Chen, "Circuit analysis and feedback amplifier theory". CRC Press, 2005.

[12]M. Singh, S. K. Roy, and B. P. Singh, "On Demand Realization of Input and Output Resistances of MOSFET Amplifier". In American Institute of
Physics Conference Proceedings, vol. 1414, no. 1, pp. 266-270. AIP, 2011.

[13]B. P, Singh, "A Null Method for Measuring the Parameters of a FET", Int. J. Electronics, Vol.44, pp.251-256, Feb.1978, ISSN: 0020-7217.

[14] W.L Everitt, and GE Anner, "Communication Engineering", McGraw-Hill, 1937.

[15] S.Darlington, "A history of network synthesis and filter theory for circuits composed of resistors, inductors, and capacitors", IEEE Trans. Circuits and Systems, vol 31, pp3-13, 1984.

[16]P. Vizmuller, "RF Design Guide: Systems, Circuits, and Equations, Artech House, 1995, ISBN 0-89006754-6.

[17]D. Jurišić, G. S. Moschytz, N. Mijat Faculty of Electrical Engineering and Computing Unska 3 Zagreb 10000 , croatia, "low-sensitivity sab bandpass active- $R C$ Filter using impedance tapering", 0 7803-6685-9/01/@2001 IEEE.

Contribution of Individual Authors to the Creation of a Scientific Article (Ghostwriting Policy)

The authors equally contributed in the present research, at all stages from the formulation of the problem to the final findings and solution.

\section{Sources of Funding for Research Presented in a Scientific Article or Scientific Article Itself}

No funding was received for conducting this study.

\section{Conflict of Interest}

The authors have no conflicts of interest to declare that are relevant to the content of this article.

Creative Commons Attribution License 4.0 (Attribution 4.0 International, CC BY 4.0)

This article is published under the terms of the Creative Commons Attribution License 4.0 https://creativecommons.org/licenses/by/4.0/deed.en 